\title{
Myocardial Perfusion Imaging with a Solid-State Camera: Simulation of a Very Low Dose Imaging Protocol
}

\author{
Ryo Nakazato ${ }^{1}$, Daniel S. Berman ${ }^{1,2}$, Sean W. Hayes ${ }^{1}$, Mathews Fish ${ }^{3}$, Richard Padgett ${ }^{4}$, Yuan Xu ${ }^{1}$, Mark Lemley ${ }^{3}$, \\ Rafael Baavour ${ }^{5}$, Nathaniel Roth ${ }^{5}$, and Piotr J. Slomka ${ }^{1,2}$ \\ ${ }^{I}$ Departments of Imaging and Medicine, and Cedars-Sinai Heart Institute, Cedars-Sinai Medical Center, Los Angeles, California; \\ ${ }^{2}$ David Geffen School of Medicine, University of California Los Angeles, Los Angeles, California; ${ }^{3}$ Oregon Heart and Vascular \\ Institute, Sacred Heart Medical Center, Springfield, Oregon; ${ }^{4}$ Oregon Heart and Vascular Institute, Sacred Heart Medical Center, \\ Oregon Cardiology, Springfield, Oregon; and ${ }^{5}$ Spectrum Dynamics, Caesarea, Israel
}

High-sensitivity dedicated cardiac camera systems provide an opportunity to lower the injected doses for SPECT myocardial perfusion imaging (MPI), but the exact limits for lowering doses have not been determined. List-mode data acquisition allows for reconstruction of various fractions of acquired counts, enabling a simulation of gradually lower administered dose. We aimed to determine the feasibility of very low dose MPI by exploring the minimal count level in the myocardium required for accurate MPI. Methods: Seventy-nine patients were studied (mean body mass index, $30.0 \pm 6.6$; range, 20.2-54.0 kg/m²) who underwent 1-d standard-dose 99mTcsestamibi exercise or adenosine rest-stress MPI for clinical indications using a cadmium-zinc-telluride dedicated cardiac camera. The imaging time was $14 \mathrm{~min}$, with averaged $803 \pm$ $200 \mathrm{MBq}(21.7 \pm 5.4 \mathrm{mCi})$ of $99 \mathrm{mTc}$ injected at stress. To simulate clinical scans with a lower dose at that imaging time we reframed the list-mode raw data. Accordingly, 6 stressequivalent datasets were reconstructed containing various count fractions of the original scan. Automated quantitative perfusion and gated SPECT software was used to quantify total perfusion deficit (TPD) and ejection fraction for all 553 datasets $(7 \times 79)$. The minimal acceptable left ventricular region counts were determined on the basis of a previous report with repeatability of same-day, same-injection Anger camera studies. Pearson correlation coefficients and the SD of differences in TPD for all scans were calculated. Results: The correlations of quantitative perfusion and function analysis were excellent for both global and regional analysis between original scans and all simulated low-count scans (all $r \geq 0.95, P<0.0001$ ). The minimal acceptable counts were determined to be 1.0 million for the left ventricular region. At this count level, the SD of differences was $1.7 \%$ for TPD and $4.2 \%$ for ejection fraction. This count level would correspond to a $92.5-\mathrm{MBq}(2.5-\mathrm{mCi})$ injected dose for the 14-min acquisition or $125.8-\mathrm{MBq}(3.4-\mathrm{mCi})$ injected dose for the 10-min acquisition. Conclusion: 1.0 million counts appear to be sufficient to produce myocardial images that agree well with 8.0-million-count images on quantitative perfusion and function parameters. With a dedicated cardiac

Received Jun. 28, 2012; revision accepted Sep. 25, 2012.

For correspondence or reprints contact: Piotr J. Slomka, Artificial Intelligence in Medicine Program, Cedars-Sinai Medical Center, 8700 Beverly Blvd., Taper Building, Ste. A047, Los Angeles, CA 90048.

E-mail: slomkap@cshs.org

Published online Jan. 15, 2013.

COPYRIGHT (C 2013 by the Society of Nuclear Medicine and Molecular Imaging, Inc. camera, these images can be obtained over 10 min with an effective radiation dose of less than $1 \mathrm{mSv}$ without significant sacrifice of accuracy.

Key Words: myocardial perfusion imaging; radiation dose; cadmium-zinc-telluride; simulation study; quantification

J Nucl Med 2013; 54:373-379

DOI: 10.2967/jnumed.112.110601

$\mathbf{R}$ educing patient radiation exposure has become an important challenge for nuclear cardiology. The last few years brought several rapid developments that have increased photon sensitivity in nuclear cardiology scanner hardware. Additionally, software applying new methods of SPECT reconstruction on conventional and dedicated systems has preserved or even improved SPECT image quality with lower count statistics (1-9). As a result, the acquisition time for SPECT myocardial perfusion imaging (MPI) may be significantly shortened. Several new dedicated camera systems with optimized acquisition geometry and collimator design resulting in increased count sensitivity have been introduced by vendors (10-16). Although it is convenient for patients to have imaging completed in 2-4 min, the efficiency of these systems provides the opportunity to reduce both the duration of the acquisition and the radiation dose to the patient. The degree to which the radiation dose can be reduced while the acquisition times are simultaneously reduced has not been explored.

List-mode data acquisition available in these new systems allows for reconstruction of various fractions of acquired counts such that lower levels of administered dose can be simulated. We aimed to determine, for a dedicated cardiac system with cadmium-zinc-telluride (CZT) detectors, the minimal count level in the left ventricular (LV) region that could provide reproducibility similar to a standard Anger camera. We focused on stress MPI data to simulate the minimal LV counts in a stress scan, with application to very low dose stress-only protocols (17). 


\section{MATERIALS AND METHODS}

\section{Study Patients}

We evaluated 79 consecutive patients who underwent MPI using a CZT camera (D-SPECT; Spectrum Dynamics). Patients were referred for evaluation by rest-stress MPI for clinical indications. Before scanning, we collected information on the presence of categoric cardiac risk factors in each individual. Hypertension was defined on the basis of a documented history of hypertension or treatment with antihypertensive medications. Diabetes mellitus was defined on the basis of a clinical history of diabetes or use of insulin or oral hypoglycemic agents. Dyslipidemia was also defined on the basis of clinical history or use of lipid-lowering medications. A history positive for smoking was defined as current smoking or smoking cessation less than 3 mo before MPI testing. A family history of coronary artery disease was defined as myocardial infarction or cardiac death before age $55 \mathrm{y}$ in a man and before age $65 \mathrm{y}$ in a woman as reported by the patient. The occurrence of a prior myocardial infarction was based on either an initial report by the patient or chart documentation. This study was conducted according to guidelines of the Institutional Review Board of the Oregon Heart and Vascular Institute. All patients provided written informed consent for the use of their clinical and imaging data for research purposes.

\section{Patient Preparation and Image Acquisition}

The patients were instructed to abstain from any products containing caffeine for $24 \mathrm{~h}$ before the test. $\beta$-blockers and calciumchannel antagonists were discontinued for $48 \mathrm{~h}$, and nitrates for $6 \mathrm{~h}$, before testing. Rest-stress 1-d ${ }^{99 m}$ Tc-sestamibi imaging was performed on all patients using weight-adjusted doses of 111-296 $\mathrm{MBq}$ (3-8 $\mathrm{mCi}$ ) for rest and 481-1,221 MBq (13-33 $\mathrm{mCi}$ ) for stress (12), in the upright position. Rest images were not used in this study because we focused on simulations that could be applicable to stress-only protocols (17-19). Patients underwent symptomlimited standard exercise treadmill testing $(51 \%)$ or adenosine stress $(49 \%, 140 \mu \mathrm{g} / \mathrm{kg} / \mathrm{min}$ for $6 \mathrm{~min})$ with injection at peak stress $(20,21)$. Stress imaging began at 15-30 min after injection and continued for $14 \mathrm{~min}$. With regard to the administered dose of ${ }^{99} \mathrm{~m} \mathrm{Tc}$-sestamibi, in our experience we have found that an average of $74 \mathrm{MBq}(2 \mathrm{mCi})$ remains in the syringe and the injection tubing.

A full description of the dedicated cardiac system used in this study has been previously reported $(13,22,23)$. Briefly, the system uses 9 tungsten collimated CZT detector columns rotating in synchrony, each consisting of 1,024 $(16 \times 64)$, 5-mm-thick CZT elements $(2.46 \times 2.46 \mathrm{~mm})$. The size of the collimator holes matches the dimensions of the detector elements. Before imaging, the detector was positioned parallel to the patient's chest, with the heart in the center of the field of view. A 6- to 12-s prescan acquisition was performed to locate the heart and to set the angle limits of scanning for each detector column (region-of-interest-centric scanning). Each image set was acquired with 120 projections per detector.

The vendor reconstruction includes 2 steps. A first reconstruction step uses a uniform gray starting image with 3 iterations. In this step, the LV region is determined for the LV count calculation. This approximate LV region reconstruction is subsequently used as a "prior" for further reconstruction of full image data with an additional 4 iterations. This final transaxial image is generated from list-mode data by the vendor reconstruction algorithm based on the maximum-likelihood expectation maximization method (23) with resolution recovery using a total of 4-7 iterations and 32 subsets and additional interiterative kernel convolution smoothing (12).
These parameters are the default reconstruction parameters validated in a previous study (12) in which image quality and quantitative parameters were compared with conventional MPI. No attenuation or scatter correction was applied. Images were then automatically reoriented into short-axis and vertical and horizontal long-axis slices using standard quantitative perfusion SPECT (QPS) software (Cedars-Sinai Medical Center).

\section{Count Determination}

The LV counts were determined from the planar projections of the approximate LV region defined during the first step of data reconstruction. We could not use the reconstructed image data and accurate 3-dimensional LV segmentation, since the vendor adjusts the reconstructed counts to account for the efficiency of the detectors and collimators. During this first reconstruction step, a circular region of interest was adjusted by the observer around the LV on the transaxial, coronal, and sagittal projections. Subsequently, the volumetric region of interest was back-projected on all the detectors, creating planar regions of interest, which were used for the LV count calculation. In this way, most of the extracardiac and background activity was eliminated.

\section{Simulation of Lower Injected Dose}

To simulate a clinical scan with lower counts acquired over the full acquisition time, the patient's list-mode raw data were reframed such that only a fraction of each view was used, and subsequently these truncated views were reconstructed in the same manner as for the full-count scans. For example, a 14-min, 120-view scan acquired with $7 \mathrm{~s}$ per view was reframed to a subset of the data with $3.5 \mathrm{~s}$ per view to simulate half-dose images (Fig. 1). Using this methodology, 6 low-count stress scans were reconstructed from each full-count reconstruction scan to simulate various levels of myocardial counts (3.6, 2.0, 1.3, 1.0, 0.7, and 0.5 million counts [MC]). The acquisition and reconstruction parameters were the same for all data used in the current study.

\section{Image Analysis}

Automated QPS and quantitative gated SPECT (QGS; CedarsSinai Medical Center) software was used to quantify myocardial perfusion and function for all 553 datasets $(7 \times 79)(24)$. The quantitative perfusion variable used was total perfusion deficit (TPD), which reflects a combination of both defect severity and the extent of the defect in a single parameter, as previously described $(25,26)$. On a per-patient basis, abnormal stress TPD was

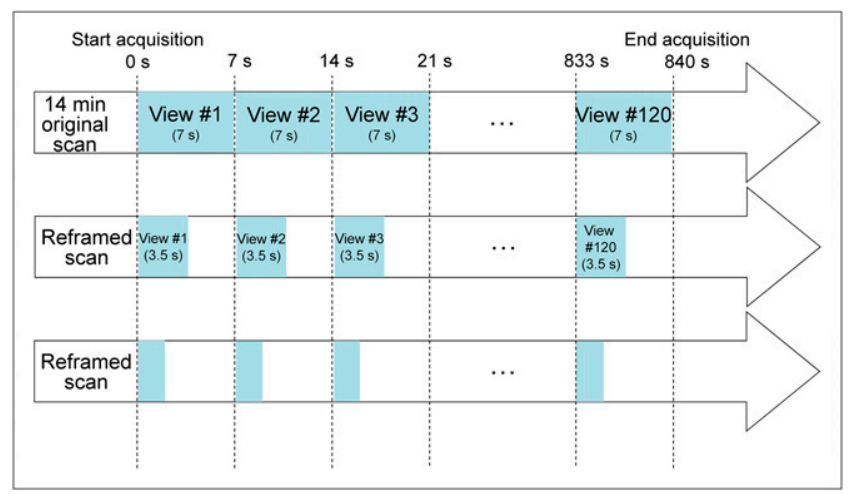

FIGURE 1. Reframing methodology: a time reframing for each view. Each CZT-SPECT clinical scan is done with 120 views generally between 1 and $7 \mathrm{~s}$ per view. By selecting part of each view, we can simulate reduction in scanning time retrospectively. 
defined as being at least 5\% and was categorized as mild (5\%-10\%) or moderate/severe ( $>10 \%$ ) (27). Regional TPD was also calculated for each vascular territory, with abnormal being defined as at least $2 \%$ (26). We analyzed these cases separately to assess the minimal counts required in patients with abnormal TPD.

From this population, we generated count-specific reference normal limits using 34 patients ( 14 men and 20 women) who had normal findings on rest and stress images by visual assessment (26). Stress perfusion defects were assessed by quantification of TPD using these count-specific normal limits as well as standard limits previously obtained for this system (26). LV ejection fraction was quantified using a modified version of QGS (28) accounting for higher resolution and better visibility of the myocardial base (29).

\section{Statistical Analysis}

Continuous variables are presented as means \pm SDs and categoric variables as frequencies. Pearson correlation coefficient, Bland-Altman limits of agreement, and SD of differences were calculated using a high-count study of $8.0 \mathrm{MC}$ as the reference standard. A 2-tailed $P$ value of less than 0.05 was considered statistically significant. The minimal acceptable count was based on a previous report with repeatability of same-day, same-injection Anger camera studies (30). All statistical calculations were performed using STATA (version 11; StataCorp LP) for Windows (Microsoft).

\section{RESULTS}

The patient characteristics of the study population are given in Table 1. Mean body mass index (BMI) was $30.0 \pm$ $6.6 \mathrm{~kg} / \mathrm{m}^{2}$, and the range was $20.2-54.0 \mathrm{~kg} / \mathrm{m}^{2}$. Thirty-two of 79 patients had abnormal stress TPD $(\geq 5 \%)$, including 12 patients with mild stress perfusion defects (TPD of 5\%$10 \%)$ and 20 patients with moderate/severe stress perfusion defects (TPD $>10 \%)$. In 32 patients with abnormal stress TPD, 8 had fixed perfusion defects, and 24 had reversible perfusion defects in the 8.0-MC scan. On a per-vessel analysis, stress perfusion abnormality (TPD $\geq 2 \%$ per vascular territory) was seen in 22, 22, and 19 patients in the left anterior descending, left circumflex, and right coronary artery territories, respectively. A mean dose of $802.9 \pm 199.8 \mathrm{MBq}$

TABLE 1

Patient Characteristics $(n=79)$

\begin{tabular}{lc}
\hline \multicolumn{1}{c}{ Characteristic } & Value \\
\hline Male $(n)$ & $38(48 \%)$ \\
Mean age \pm SD $(\mathrm{y})$ & $65 \pm 10$ \\
$\mathrm{BMI}\left(\mathrm{kg} / \mathrm{m}^{2}\right)$ & \\
$\quad$ Mean $\pm \mathrm{SD}$ & $30.0 \pm 6.6$ \\
$\quad$ Range & $20.2-54.0$ \\
Hypertension $(n)$ & $50(63 \%)$ \\
Dyslipidemia $(n)$ & $53(67 \%)$ \\
Diabetes $(n)$ & $19(24 \%)$ \\
Current smoking $(n)$ & $13(16 \%)$ \\
Family history of coronary artery disease $(n)$ & $30(38 \%)$ \\
Previous myocardial infarction $(n)$ & $14(18 \%)$ \\
Previous percutaneous coronary intervention $(n)$ & $12(15 \%)$ \\
Previous coronary artery bypass graft $(n)$ & $15(19 \%)$ \\
Exercise stress $(n)$ & $40(51 \%)$ \\
\hline
\end{tabular}

$(21.7 \pm 5.4 \mathrm{mCi})$ of ${ }^{99 \mathrm{~m}} \mathrm{Tc}-$ sestamibi for stress was drawn up in the syringe; assuming $74 \mathrm{MBq}(2 \mathrm{mCi})$ remained in the syringe and the injection tubing, $728.9 \mathrm{MBq}(19.7 \mathrm{mCi})$ were administered to the patients. The average LV counts for the stress scan were 7,962,719 $\pm 2,349,989,3,598,744 \pm$ $14,929,2,000,125 \pm 7,092,1,300,958 \pm 8,121,1,000,180 \pm$ $5,177,700,363 \pm 5,332$, and $500,760 \pm 3,964$ for the $8.0-$, 3.6-, 2.0-, 1.3-, 1.0-, 0.7-, and 0.5-MC scans, respectively.

An experienced imaging technologist without knowledge of the quantitative results determined that 14 of 553 myocardial contours generated from QPS needed adjustment for static perfusion images, with no relationship to the number of myocardial counts in the study (Table 2).

The reproducibility of simulated global perfusion and function results is shown in Table 3. The correlations of quantitative perfusion analysis were excellent for global analysis in all simulated low-count scans (all $r \geq 0.99, P<0.0001$ ). At the level of 1.0 MC, the SD of the differences for TPD was $1.7 \%$, and the SD of the differences for ejection fraction was $4.2 \%$. Bland-Altman analyses for global perfusion quantification are shown in Figure 2. Compared with the 8.0-MC scan, the reduced-count studies showed a small negative bias, which increased as counts were reduced; however, the BlandAltman plots demonstrated that bias remained low through the range of simulated low-count scans. In patients with abnormal stress perfusion defects (separately evaluated), the correlation was also excellent $(r=0.99, P<0.0001)$ and the SD of differences was $1.7 \%$ at the level of $1.0 \mathrm{MC}$. In patients without abnormal stress perfusion defects, the SD of differences was $1.2 \%$ at the level of $1.0 \mathrm{MC}$. The results were similar between patients with and without abnormal stress perfusion defects. In patients with a BMI greater than $30 \mathrm{~kg} / \mathrm{m}^{2}(n=34)$, the correlation was also excellent $(r=$ $0.99, P<0.0001)$ and the SD of differences was $1.4 \%$ at the level of 1.0 MC.

Figure 3 shows the bias of TPD values using the countspecific normal limits compared with using the same normal limits for different levels of counts. The use of the countspecific normal limits was associated with a significant reduction of TPD bias from $1.4 \%$ to $0.6 \%$ in the overall population. Similarly, in patients with a BMI greater than $30 \mathrm{~kg} / \mathrm{m}^{2}$, TPD bias was $22 \%$ lower on average using the count-specific normal limits.

TABLE 2

Number of Cases Requiring Contour Adjustment

\begin{tabular}{lcc}
\hline Counts $(\mathrm{MC})$ & Static Adjusted & Gate Adjusted \\
\hline 8.0 (reference) & $1(1 \%)$ & $3(4 \%)$ \\
3.6 & $4(5 \%)$ & $4(5 \%)$ \\
2.0 & $2(3 \%)$ & $5(6 \%)$ \\
1.3 & $2(3 \%)$ & $6(8 \%)$ \\
1.0 & $1(1 \%)$ & $5(6 \%)$ \\
0.7 & $2(3 \%)$ & $4(5 \%)$ \\
0.5 & $2(3 \%)$ & $6(8 \%)$ \\
\hline
\end{tabular}


TABLE 3

Global Reproducibility of Perfusion and Function Parameters

\begin{tabular}{lcccc}
\hline Counts (MC) & Correlation (TPD) & SD of differences (TPD) & Correlation (ejection fraction) & SD of differences (ejection fraction) \\
\hline 8.0 (reference) & Reference & Reference & Reference \\
3.6 & 1.00 & 0.9 & 0.98 \\
2.0 & 0.99 & 1.1 & 0.98 \\
1.3 & 0.99 & 1.4 & 0.96 \\
1.0 & 0.99 & 1.7 & 0.97 & 3.1 \\
0.7 & 0.99 & 2.0 & 0.96 & 4.2 \\
0.5 & 0.99 & 2.2 & 0.96 & 4.2 \\
\multicolumn{2}{l}{ Count-specific normal limits were used for TPD. }
\end{tabular}

The reproducibility of simulated perfusion results for regional perfusion analysis is shown in Table 4. Correlation values were similar to those observed for global perfusion analysis on the simulated low-count scans (all $r \geq$ $0.95, P<0.0001)$.

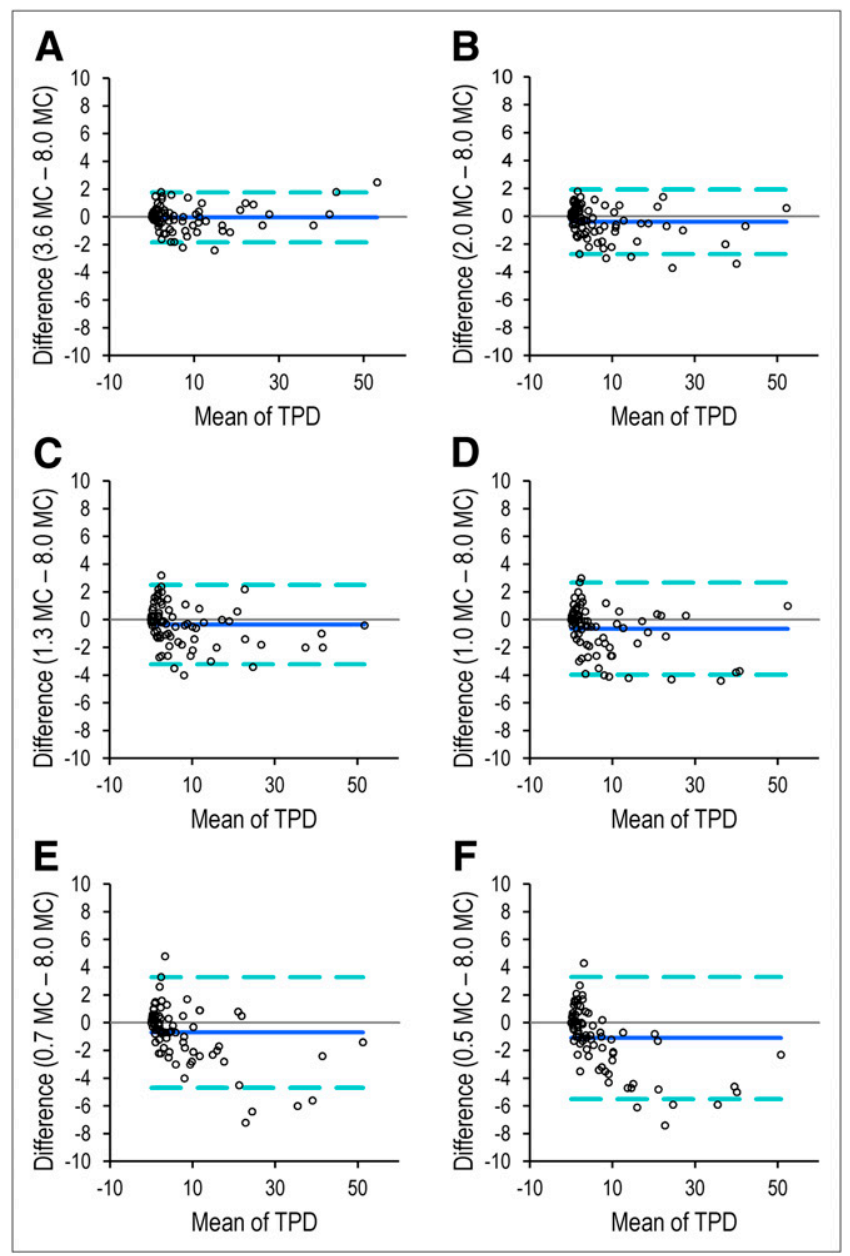

FIGURE 2. Bland-Altman plots for TPD at different count levels3.6 (A), $2.0(\mathrm{~B}), 1.3(\mathrm{C}), 1.0(\mathrm{D}), 0.7(\mathrm{E})$, and $0.5(\mathrm{~F}) \mathrm{MC}$ - vs. TPD for high-count scan (8.0 MC). Count-specific normal limits were used for TPD.
In a previous report with repeatability of same-day, sameinjection full-count Anger camera studies, the SD of the differences was $1.7 \%$ for TPD and $3.6 \%$ for ejection fraction (30). In the present study, on a 1.0-MC scan the SD of the differences was $1.7 \%$ for TPD and $4.2 \%$ for ejection fraction. There was no significant difference in the SD of the differences for ejection fraction between these 2 reports $(P=0.4)$. Therefore, the minimal acceptable count level was determined to be $1.0 \mathrm{MC}$ for stress CZT MPI. For the 14-min acquisition, the 1.0-MC scan allows the injected dose to be reduced by a factor of 8 , which corresponds to an average injected dose of $92.5 \pm$ $25.9 \mathrm{MBq}(2.5 \pm 0.7 \mathrm{mCi})$ (range, $51.8-144.3 \mathrm{MBq}[1.4-$ $3.9 \mathrm{mCi}]$ ) and would have resulted in an effective average radiation dose of $0.71 \pm 0.20 \mathrm{mSv}$ (range, 0.40 to $1.11 \mathrm{mSv}$ ) in a 14-min study and an average radiation dose of $0.99 \pm$ $0.28 \mathrm{mSv}$ (range, $0.56-1.55 \mathrm{mSv}$ ) in a 10 -min study. In patients with a BMI greater than $30 \mathrm{~kg} / \mathrm{m}^{2}$ at the level of 1.0 MC (average counts, 1,001,319), the average injected dose would be $111.0 \pm 25.9 \mathrm{MBq}(3.0 \pm 0.7 \mathrm{mCi})$ (range,

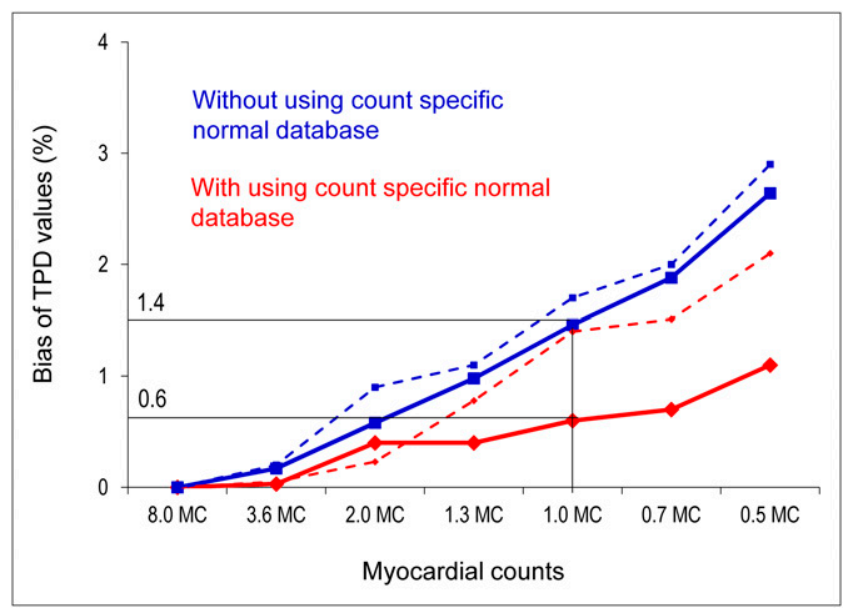

FIGURE 3. TPD bias as a function of decreasing count levels with (red) and without (blue) count-specific normal limits. Solid lines correspond to TPD bias for overall population $(n=79)$. Dashed lines correspond to TPD bias for patients with abnormal stress perfusion $(n=32)$. 
TABLE 4

Regional Reproducibility of Perfusion Parameters

\begin{tabular}{|c|c|c|}
\hline Counts (MC) & $\begin{array}{l}\text { Correlation } \\
\text { (TPD) }\end{array}$ & $\begin{array}{c}\text { SD of differences } \\
\text { (TPD) }\end{array}$ \\
\hline \multicolumn{3}{|c|}{$\begin{array}{l}\text { Left anterior } \\
\text { descending region }\end{array}$} \\
\hline 8.0 (reference) & Reference & Reference \\
\hline 3.6 & 1.00 & 0.6 \\
\hline 2.0 & 1.00 & 0.6 \\
\hline 1.3 & 0.99 & 0.8 \\
\hline 1.0 & 0.99 & 0.8 \\
\hline 0.7 & 0.99 & 0.9 \\
\hline 0.5 & 0.99 & 1.1 \\
\hline \multicolumn{3}{|l|}{$\begin{array}{l}\text { Left circumflex } \\
\text { region }\end{array}$} \\
\hline 8.0 (reference) & Reference & Reference \\
\hline 3.6 & 0.99 & 0.5 \\
\hline 2.0 & 0.99 & 0.6 \\
\hline 1.3 & 0.96 & 1.1 \\
\hline 1.0 & 0.97 & 1.1 \\
\hline 0.7 & 0.96 & 1.1 \\
\hline 0.5 & 0.95 & 1.3 \\
\hline \multicolumn{3}{|l|}{$\begin{array}{l}\text { Right coronary } \\
\text { artery region }\end{array}$} \\
\hline 8.0 (reference) & Reference & Reference \\
\hline 3.6 & 0.99 & 0.4 \\
\hline 2.0 & 0.99 & 0.4 \\
\hline 1.3 & 0.99 & 0.5 \\
\hline 1.0 & 0.99 & 0.5 \\
\hline 0.7 & 0.98 & 0.7 \\
\hline 0.5 & 0.96 & 1.0 \\
\hline
\end{tabular}

Count-specific normal limits were used for TPD.

66.6-144.3 $\mathrm{MBq}$ [1.8-3.9 $\mathrm{mCi}]$ ) with an average radiation dose of $0.85 \pm 0.20 \mathrm{mSv}$ (range, $0.51-1.11 \mathrm{mSv}$ ) in a 14-min study.

Case examples of images at gradually reduced doses are shown in Figures 4 and 5.

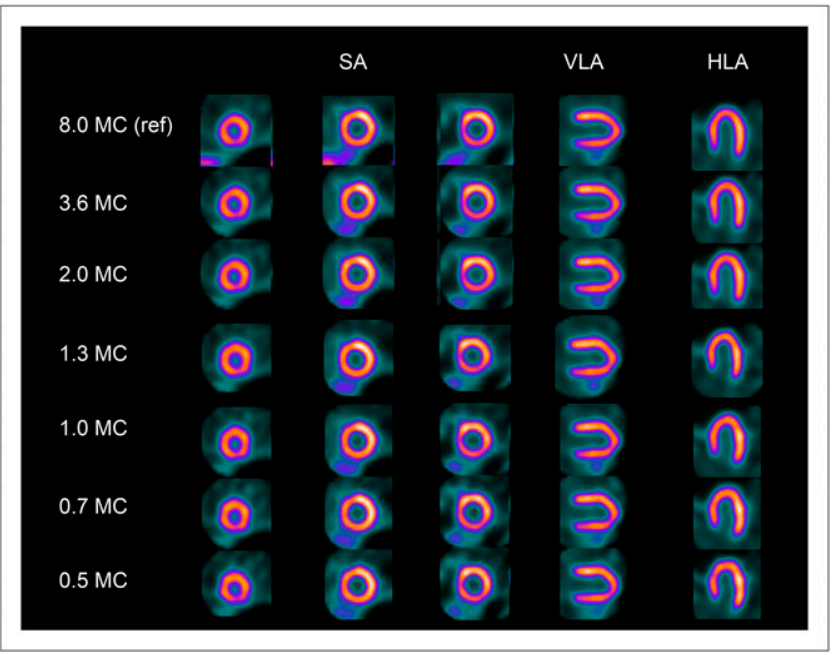

FIGURE 4. Case example of normal-perfusion images. Original and all reframed scans had TPD of $0 \%$. SA = short axis; VLA = vertical long axis; HLA = horizontal long axis.

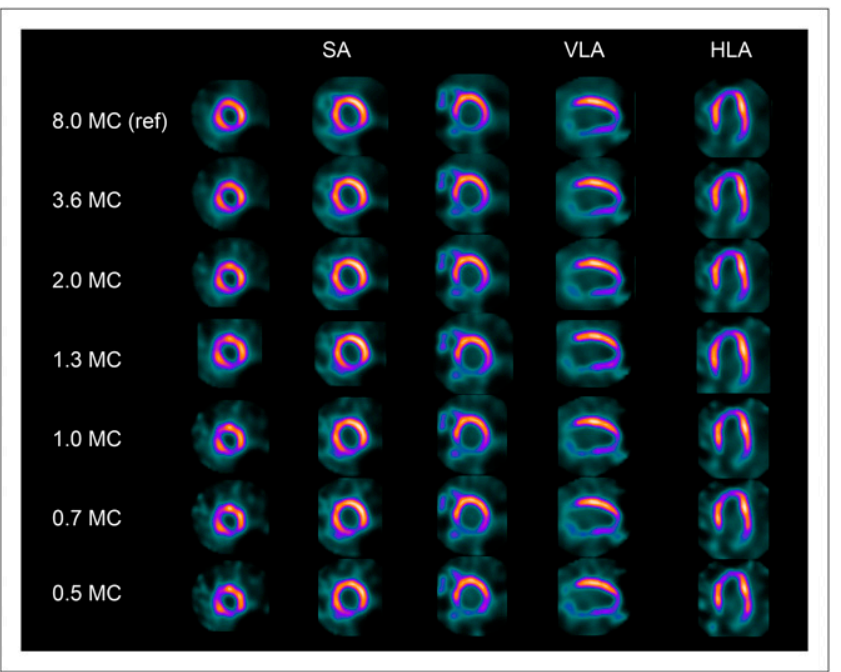

FIGURE 5. Case example of abnormal-perfusion images.TPD values were $11 \%, 12 \%, 12 \%, 12 \%, 12 \%, 12 \%$, and $9 \%$ for $8.0,3.6$, $2.0,1.3,1.0,0.7$, and $0.5 \mathrm{MC}$, respectively. HLA = horizontal long axis; $\mathrm{SA}=$ short axis; VLA $=$ vertical long axis.

\section{DISCUSSION}

Reducing the effective radiation dose to patients and reducing the exposure of staff to radiation are important goals in the field of nuclear cardiology (31). Our study demonstrated that dedicated cardiac scanners may allow acquisitions with average $\mathrm{LV}$ region counts of as low as 1.0 MC for stress MPI regardless of the presence of stress perfusion defects while maintaining excellent agreement in quantitative perfusion and function parameters with those determined from very high count images. Our calculations used the average doses administered, and the estimate specific to a given patient may vary depending on the injected dose (based on the patient's BMI). Therefore, the minimal dose could be higher in patients with a higher BMI and lower in patients with a lower BMI. For patients with a BMI greater than $30 \mathrm{~kg} / \mathrm{m}^{2}$, the average estimated radiation dose of $0.85 \pm 0.20 \mathrm{mSv}$ (range, $0.51-1.11 \mathrm{mSv}$ ) at the 1 -MC level was higher than in the overall study population $(0.71 \pm 0.20 \mathrm{mSv}$ [range, $0.40-1.11 \mathrm{mSv}$ ]).

The findings show that the amount of injected radioactivity can be reduced by a factor of 8 with a 14-min acquisition, resulting in a $1.0-\mathrm{MC}$ scan with a $92.5-\mathrm{MBq}(2.5-\mathrm{mCi})$ injected dose, which would be associated with an average $0.71-\mathrm{mSv}$ effective radiation dose to the patient (32). Because it is desirable to reduce both radiation dose and scanning time, these findings further imply that by increasing the injected dose to $125.8 \mathrm{MBq}$ ( $3.4 \mathrm{mCi})$, a submillisievert stress-only MPI study can be achieved with an acquisition time of $10 \mathrm{~min}$. However, a larger, prospective multicenter trial is warranted for further investigation.

We established precise minimum count levels by retrospective analysis of full-dose, full-time data and reconstruction of only a fraction of the counts spread over the full acquisition time. Such an approach is facilitated by the 
list-mode capabilities of the new scanners. Especially for the new stationary scanners, it is possible to retrospectively simulate a scan with an arbitrary activity or, alternatively, duration from the original full-dose, full-time acquisition data. Using a similar approach, Herzog et al. have shown by retrospective list-mode reconstruction that a 2-min acquisition is optimal for the ultrafast cardiac scanner (GE Healthcare) (14). In our study, the data were acquired over the full imaging time, and therefore the simulation should closely correspond to low-dose, full-time acquisition protocols.

The reference standard of $8.0 \mathrm{MC}$ is a very high photon count-much higher than currently obtained by Anger cameras. The reason why significant count reductions do not translate into poorer reproducibility is the high sensitivity of the collimators used and detector geometry focused on the cardiac region, rather than the use of solid-state detectors. These characteristics allow 6-8 times the counting rate of the conventional system to be obtained. Acquisitions obtained with these new systems at standard doses and standard counting rates have a very high photon count; therefore, significant reductions can be obtained while still maintaining adequate image statistics. Similar simulation studies can be performed for other dedicated cardiac systems without solid detectors (4) or for new reconstruction methods $(7,9)$ if list-mode acquisition is available. Our findings show little benefit to imaging with such high count levels, compared with low-count studies.

In the present study, we used fully automated quantitative analysis of MPI studies. Quantitative analysis eliminates observer variability and bias and is useful for comparing sequential MPI studies $(33,34)$. Quantitative analysis also provides an approach that is more generalizable to other centers, as it is not dependent on the expertise of the interpreter. The reported variability is the overall variability due to the count level differences but also to the intrinsic variability of the quantitative software (sampling methods, valve plane determination). However, this overall variability is the practical clinical measure by which low-dose imaging can be judged. We have demonstrated here that this overall variability for studies with gradually decreasing count levels ( $\leq 8$ times) is similar to the quantitative variability of repeated studies with the same count level obtained on a Anger camera.

The use of the same normal limits for all count levels (26) was associated with increasing bias in TPD values. Countspecific normal limits allowed a significant reduction of this bias. Therefore, our findings indicate that count-specific normal limits are needed to provide comparable findings for studies with different count levels, since the normal thresholds for perfusion studies can be affected by the bias.

The method used for count estimation may overestimate myocardial counts because of the approximate shape of the LV region. Although the use of 3-dimensional QGS and QPS boundaries can improve the results by excluding background activity more precisely, in the current implementation of the vendor reconstruction the tomographic counts cannot be used because they are scaled to correct for known detector and collimator efficiencies. However, the advantage of our method for LV count estimation is that it is easily available to the scanner operator, without the need for additional software processing during image acquisition. Even if 3-dimensional LV counts have been estimated from the precise LV segmentation, the myocardial count levels would still be affected by the extent of hypoperfusion. We have, however, shown good reproducibility in abnormal studies with low count levels as well.

Our study has several limitations. The acquisitions were performed at only a single center with a single type of system; however, our application of automated quantitative analysis in this study increases the likelihood that our results can be replicated elsewhere. More important, because of the specialized high-dose, full-time protocol, which was used in only a limited number of patients, we could not use a separate population for development of count-specific normal limits. However, we obtained the results separately in a population with abnormal stress perfusion defects, and these results agree with the findings in the overall population. We included patients with a BMI ranging from 20.2 to $54.0 \mathrm{~kg} / \mathrm{m}^{2}$. However, the mean BMI was $30.0 \mathrm{~kg} / \mathrm{m}^{2}$ in our population. Caution must be taken when extrapolating the average estimated dose to patients with a substantially higher BMI. Therefore, we also provided ranges of the estimated doses and separately evaluated the doses for the patients with a high BMI. We did not use attenuation correction, because this camera is not equipped with that option.

\section{CONCLUSION}

This simulation study based on reframing of patient image data suggests that a dedicated cardiac scanner may allow counts of as low as 1.0 MC for stress MPI while maintaining excellent agreement in quantitative perfusion and function parameters with those determined from highcount, full-time images. Our findings suggest that a 10-min stress scan can be performed with an effective average radiation dose of less than $1 \mathrm{mSv}$ with the dedicated cardiac scanners.

\section{DISCLOSURE}

The costs of publication of this article were defrayed in part by the payment of page charges. Therefore, and solely to indicate this fact, this article is hereby marked "advertisement" in accordance with 18 USC section 1734. This research was supported in part by grant R01-HL089765 from the National Heart, Lung, and Blood Institute/National Institutes of Health (NHLBI/NIH). The content of the article is solely the responsibility of the authors and does not necessarily represent the official views of the NHLBI. Dr. Daniel Berman is a shareholder in Spectrum-Dynamics. Rafael Baavour and Nathaniel Roth are Spectrum-Dynamics company employees. No other potential conflict of interest relevant to this article was reported. 


\section{ACKNOWLEDGMENT}

We thank Arpine Oganyan for editing and proofreading the manuscript.

\section{REFERENCES}

1. DePuey EG, Gadiraju R, Clark J, Thompson L, Anstett F, Shwartz SC. Ordered subset expectation maximization and wide beam reconstruction "half-time" gated myocardial perfusion SPECT functional imaging: a comparison to "fulltime" filtered backprojection. J Nucl Cardiol. 2008;15:547-563.

2. Borges-Neto S, Pagnanelli RA, Shaw LK, et al. Clinical results of a novel wide beam reconstruction method for shortening scan time of Tc-99m cardiac SPECT perfusion studies. J Nucl Cardiol. 2007;14:555-565.

3. Vija H, Chapman J, Ray M. IQ•SPECT Technology White Paper. Malvern, PA: Siemens Medical Solutions USA Molecular Imaging; 2008:1-7.

4. Venero CV, Heller GV, Bateman TM, et al. A multicenter evaluation of a new post-processing method with depth-dependent collimator resolution applied to full-time and half-time acquisitions without and with simultaneously acquired attenuation correction. J Nucl Cardiol. 2009;16:714-725.

5. Maddahi J, Mendez R, Mahmarian JJ, et al. Prospective multicenter evaluation of rapid, gated SPECT myocardial perfusion upright imaging. J Nucl Cardiol. 2009;16:351-357.

6. Druz RS, Phillips LM, Chugkowski M, Boutis L, Rutkin B, Katz S. Wide-beam reconstruction half-time SPECT improves diagnostic certainty and preserves normalcy and accuracy: a quantitative perfusion analysis. J Nucl Cardiol. 2011; 18:52-61.

7. DePuey EG, Bommireddipalli S, Clark J, Leykekhman A, Thompson LB, Friedman M. A comparison of the image quality of full-time myocardial perfusion SPECT vs wide beam reconstruction half-time and half-dose SPECT. $J$ Nucl Cardiol. 2011;18:273-280.

8. Depuey EG, Ata P, Wray R, Friedman M. Very low-activity stress/high-activity rest, single-day myocardial perfusion SPECT with a conventional sodium iodide camera and wide beam reconstruction processing. J Nucl Cardiol. 2012;19:931944.

9. DePuey EG, Bommireddipalli S, Clark J, Thompson L, Srour Y. Wide beam reconstruction "quarter-time" gated myocardial perfusion SPECT functional imaging: a comparison to "full-time" ordered subset expectation maximum. J Nucl Cardiol. 2009; 16:736-752.

10. Slomka PJ, Patton J, Berman D, Germano G. Advances in technical aspects of myocardial perfusion SPECT imaging. J Nucl Cardiol. 2009;16:255-276.

11. Garcia EV, Faber TL, Esteves FP. Cardiac dedicated ultrafast SPECT cameras: new designs and clinical implications. J Nucl Med. 2011;52:210-217.

12. Sharir T, Slomka P, Hayes S, et al. Multicenter trial of high-speed versus conventional single-photon emission computed tomography imaging: quantitative results of myocardial perfusion and left ventricular function. J Am Coll Cardiol. 2010;55:1965-1974.

13. Sharir T, Ben-Haim S, Merzon K, Prochorov V, Dickman D, Berman D. Highspeed myocardial perfusion imaging initial clinical comparison with conventional dual detector Anger camera imaging. JACC Cardiovasc Imaging. 2008;1: 156-163.

14. Herzog BA, Buechel RR, Katz R, et al. Nuclear myocardial perfusion imaging with a cadmium-zinc-telluride detector technique: optimized protocol for scan time reduction. J Nucl Med. 2010;51:46-51.

15. Buechel RR, Herzog BA, Husmann L, et al. Ultrafast nuclear myocardial perfusion imaging on a new gamma camera with semiconductor detector technique: first clinical validation. Eur J Nucl Med Mol Imaging. 2010;37:773-778.
16. Esteves FP, Raggi P, Folks RD, et al. Novel solid-state-detector dedicated cardiac camera for fast myocardial perfusion imaging: multicenter comparison with standard dual detector cameras. J Nucl Cardiol. 2009;16:927-934.

17. Bateman T, McGhie A, Courter S, Burgett E, Cullom J, Case J. Prospective study of ultra-low dose stress only solid-state SPECT: comparison of efficiency, dosimetry and outcome versus traditional-dose attenuation-corrected stress-only Anger SPECT (NCT01373944) [abstract]. J Am Coll Cardiol. 2012;59(suppl): E1316.

18. Duvall WL, Wijetunga MN, Klein TM, et al. The prognosis of a normal stressonly Tc-99m myocardial perfusion imaging study. J Nucl Cardiol. 2010;17:370377.

19. Chang SM, Nabi F, Xu J, Raza U, Mahmarian JJ. Normal stress-only versus standard stress/rest myocardial perfusion imaging: similar patient mortality with reduced radiation exposure. J Am Coll Cardiol. 2010;55:221-230.

20. Berman DS, Abidov A, Kang X, et al. Prognostic validation of a 17-segment score derived from a 20-segment score for myocardial perfusion SPECT interpretation. J Nucl Cardiol. 2004;11:414-423.

21. Berman D, Germano G. Interpretation and reporting of gated myocardial perfusion SPECT. In: Germano G, Berman DS, eds. Clinical Gated Cardiac SPECT. 2nd ed. Oxford, U.K.: Blackwell Publishing; 2006:139-171.

22. Patton JA, Slomka P, Germano G, Berman D. Recent technologic advances in nuclear cardiology. J Nucl Cardiol. 2007;14:501-513.

23. Gambhir SS, Berman D, Ziffer J, et al. A novel high-sensitivity rapid-acquisition single-photon cardiac imaging camera. J Nucl Med. 2009;50:635-643.

24. Germano G, Kavanagh PB, Slomka PJ, Van Kriekinge SD, Pollard G, Berman DS. Quantitation in gated perfusion SPECT imaging: the Cedars-Sinai approach. J Nucl Cardiol. 2007;14:433-454.

25. Slomka PJ, Nishina H, Berman D, et al. Automated quantification of myocardial perfusion SPECT using simplified normal limits. J Nucl Cardiol. 2005;12:66-77.

26. Nakazato R, Tamarappoo BK, Kang X, et al. Quantitative upright-supine highspeed SPECT myocardial perfusion imaging for detection of coronary artery disease: correlation with invasive coronary angiography. J Nucl Med. 2010;51: 1724-1731.

27. Hachamovitch R, Hayes S, Friedman J, Cohen I, Berman D. Comparison of the short-term survival benefit associated with revascularization compared with medical therapy in patients with no prior coronary artery disease undergoing stress myocardial perfusion single photon emission computed tomography. Circulation. 2003;107:2900-2907.

28. Germano G, Kiat H, Kavanagh PB, et al. Automatic quantification of ejection fraction from gated myocardial perfusion SPECT. J Nucl Med. 1995;36:21382147.

29. Slomka P, Germano G, Kavanagh P, Javadi M, Berman D, Bengel F. Evaluation of a new automatic algorithm for quantification of ECG-gated ${ }^{82} \mathrm{Rb}$ cardiac PET [abstract]. J Nucl Med. 2009;50(suppl 2):217P.

30. Xu Y, Hayes S, Ali I, et al. Automatic and visual reproducibility of perfusion and function measures for myocardial perfusion SPECT. J Nucl Cardiol. 2010;17: 1050-1057.

31. Cerqueira MD, Allman KC, Ficaro EP, et al. Recommendations for reducing radiation exposure in myocardial perfusion imaging. J Nucl Cardiol. 2010;17: $709-718$.

32. Einstein AJ, Moser KW, Thompson RC, Cerqueira MD, Henzlova MJ. Radiation dose to patients from cardiac diagnostic imaging. Circulation. 2007;116:12901305 .

33. Berman DS, Kang X, Gransar H, et al. Quantitative assessment of myocardial perfusion abnormality on SPECT myocardial perfusion imaging is more reproducible than expert visual analysis. J Nucl Cardiol. 2009;16:45-53.

34. Slomka PJ, Berman D, Germano G. Quantification of serial changes in myocardial perfusion. J Nucl Med. 2004;45:1978-1980. 